\title{
LE MURA SPAGNOLE DI MILANO: GLI INTERVENTI CONSERVATIVI (2006-2009) E LA LORO NEGAZIONE
}

\author{
REBECCA FANT (*) \\ Nota presentata dal m.e. Amedeo Bellini \\ (Adunanza del 1 Marzo 2012)
}

SuNTO. - In questo lavoro si presenta l'approccio metodologico conservativo applicato all'intervento di restauro, che nasce da un percorso di conoscenza e valorizzazione dei resti del sistema difensivo delle Mura Spagnole. Tale approccio deriva da un riconoscimento del valore testimoniale, nei secoli scorsi negato, e dall'applicazione di indagini e attenzione al dato materiale che hanno indirizzato il progetto esecutivo verso metodologie più corrette. Il vero problema della permanenza materiale del monumento però si pone nell'ottica della "conservazione preventiva e programmata", ovvero della cura costante, del passaggio da una fase di "correzione", prevista con l'intervento di restauro, alla fase di "mantenimento". Questa fase ancora oggi risulta negata.

$$
* * *
$$

ABSTRACT. - In this paper is presented the methodological approach to the conservation works of the Spanish defensive walls. The approach arise from a path of knowledge and valorisation, deriving its meaning from the witnessing value; this latter, being denied during the past centuries, applies to surveys and pays attention to materials data, which addressed the conservation project towards more correct methodologies. The problem of the monument material persistence applies in the direction of "planned conservation", that is a constant care, passing form a "correction" phase, foreseen with the restoration works, to a "maintenance" phase. Such a phase is currently still denied.

(*) Architetto specialista in Restauro dei Monumenti. Docente a contratto del Politecnico di Milano, Italy.

E-mail: fant@rebeccafantarchitetto.it 


\section{INTRODUZIONE}

Perché sono state restaurate le Mura spagnole?

In realtà l'incarico del rilievo in previsione di un intervento di restauro (2001) e la progettazione definitiva per i lavori "di restauro, consolidamento e recupero all'uso delle Mura Spagnole nei tratti di viale Filippetti, Beatrice d'Este, piazza Medaglie D'oro e di viale Caldara" da parte dell'arch. Pietro Ripa (2003) sono stati determinati da "cedimenti della cortina muraria" in via Filippetti che hanno costretto l'Amministrazione Comunale a porre una "cesata protettiva della salute pubblica". ${ }^{1}$ Questo progetto definitivo prevedeva un'ipotesi complessiva ripartita in due lotti: il primo lotto riguardante la struttura muraria e il secondo lotto la sistemazione delle aree a verde circostanti. Di questo progetto solamente il primo lotto sarà oggetto del bando di gara per "l'Affidamento mediante procedura di sponsorizzazione per la progettazione esecutiva e l'esecuzione dei lavori di restauro, consolidamento e recupero all'uso delle Mura Spagnole nei tratti di viale Filippetti, Beatrice d'Este, Piazza Medaglie D'oro, viale Caldara". Nel marzo del 2006 la società T.M.C. Pubblicità S.r.l., a seguito dell'aggiudicazione della gara, stipulava una Convenzione con la Direzione Centrale Tecnica - Settore Edilizia Patrimoniale e Demaniale del Comune di Milano e fin da subito sono state eseguite le indagini diagnostiche, gli studi e gli approfondimenti per la conoscenza dello stato di conservazione del manufatto, e la campionatura degli interventi, al fine di redigere il progetto esecutivo ${ }^{2}$ (Tab. 1).

Inoltre, a seguito del montaggio del ponteggio sull'Arco di Porta Romana, era stato indicato nell'autorizzazione del settembre 2006 che

1 P. RIPA (a cura di), Fascicolo n. 1, Relazione storica e descrittiva progettuale dell'intervento. Progetto definitivo. Il restauro, consolidamento e recupero all'uso delle mura spagnole "per un parco della ritrovata cinta difensiva delle mura spagnole", marzo 2003, p. 3 .

2 Per l'intervento di restauro, argomento del presente contributo, si rimanda alla documentazione di progetto depositata presso gli Enti di controllo: Comune di Milano e Soprintendenza ai Beni Architettonici e del Paesaggio di Milano; unico lavoro approfondito sull'argomento risulta la tesi di Specializzazione di A. MASTRONARDI, Il progetto di conservazione delle Mura spagnole e di Porta Romana a Milano: aspetti teorici ed operativi, Scuola di specializzazione in Beni Architettonici e del Paesaggio del Politecnico di Milano, Relatore Prof. A. BELLINI e Correlatore R. FANT, A.A. 20072008. Negli anni 2007-2009 sono stati fatti convegni e visite guidate, di cui resta traccia anche in www.milanoneicantieridellarte.it. 
“...l'intervento richiesto sull'Arco da parte della Soprintendenza ai Beni Architettonici e del Paesaggio di Milano va necessariamente attuato"; quindi è stato richiesto e predisposto anche il progetto definitivo-esecutivo per il Restauro dell'Arco di Porta Romana, sito in prossimità del Bastione di piazza Medaglie d'Oro, i cui lavori sono stati realizzati tra il 2008 e il 2009.

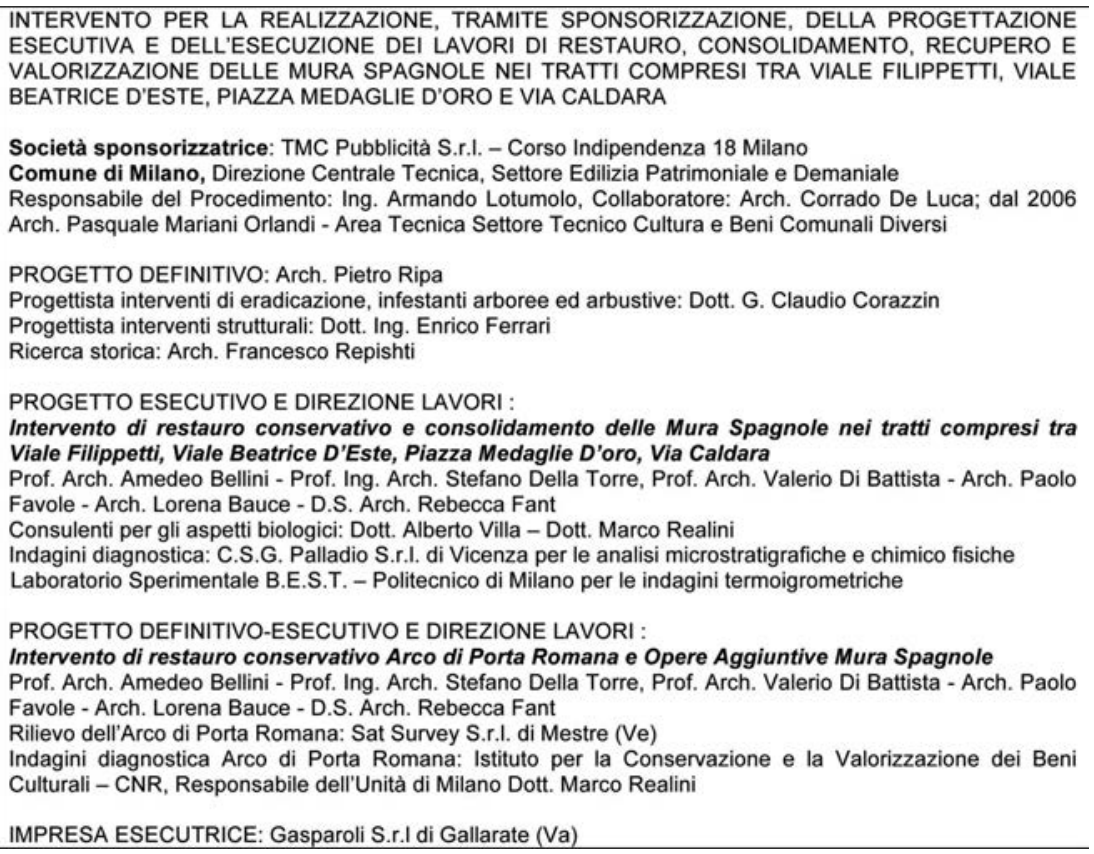

Tab. 1 - Filiera di cantiere.

Intervenire sulle Mura Spagnole ha implicato il cercare di comprendere e riconoscere le peculiarità di questo ambiente, dei resti di una architettura fortificata e delle sue vicende e trasformazioni, ma soprattutto il confrontarsi con la sua connotazione nel contesto urbano contemporaneo. Parlare oggi delle Mura Spagnole significa parlare di pochi tratti rispetto alla loro lunghezza originaria che copriva un perimetro di circa 11 chilometri. Costruite in una decina di anni a partire dal 1549, sotto la direzione dell'ingegnere Gian Maria Olgiati e per iniziativa del governatore Ferrante Gonzaga, la nuova cinta bastionata raccordava al Castello Sforzesco includendo i borghi esterni. Tali mura 
"alla moderna" costituivano un'opera militare di grandezza solenne ed erano all'epoca della loro costruzione le più imponenti d'Europa, insieme a quelle di Anversa, anche se prive di decorazioni monumentali in corrispondenza degli ingressi, almeno fino al $1598 .^{3}$ La concezione simbolica risulta ben rappresentata a partire dalla stampa cartografica prodotta da Nunzio Galizia (Fig. 1) e a seguire in tutte quelle realizzate tra il Cinquecento e il Settecento. ${ }^{4}$

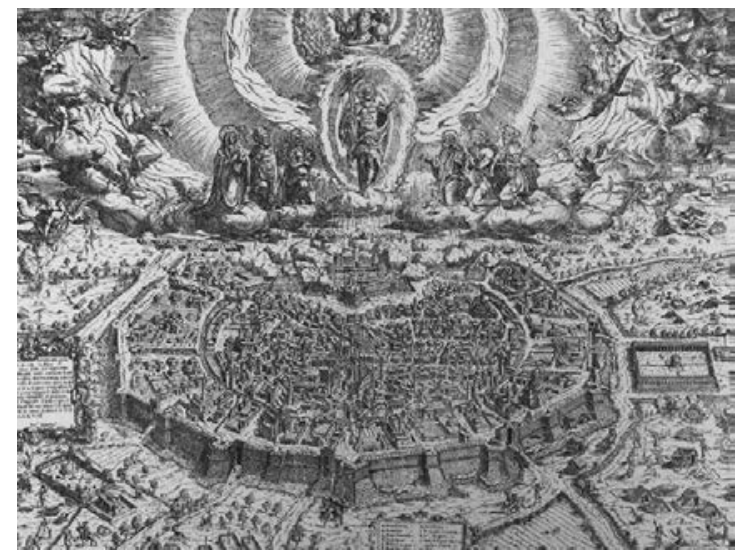

Fig. 1 - Nunzio Galizia, Milano liberata dalla peste, 1578.

3 Per le vicende storiche si vedano principalmente: A. SCOTTI, Per un profilo dell'architettura milanese (1535-1565), in Omaggio a Tiziano. La cultura artistica milanese nell' età di Carlo V, catalogo della mostra, Milano 1977, pp. 100-101; S. LEYDI, Le cavalcate dell'ingegnero. L'opera di Gianmaria Olgiati, ingegnere militare di Carlo V, ModenaFerrara, 1989; A. FILASETA, I Bastioni Spagnoli: organizzazione di cantiere e maestranze dal 1548 al 1553, in Milano città fortificata, vent'anni dopo, Atti del convegno (Milano, 1 ottobre 2003), Quaderni del Castello Sforzesco, 5, Milano 2005, pp. 48-63.

4 All'interno dell'Associazione Temporanea tra Professionisti del gruppo di progettazione, le vicende storiche sono state curate soprattutto per la parte cinquecentesca dal Prof. Stefano Della Torre e per gli interventi successivi dalla sottoscritta e dall'Arch. Lorena Bauce, insieme ad Antonella Mastronardi. Per le dettagliate vicende storiche di Porta Romana si rimanda a quanto prodotto nella relazione storica in parte ripresa in questa sede, alla bibliografia particolareggiata e alle seguenti fonti documentarie: Archivio Storico del Comune di Milano (ASCM), Località milanesi, 281; Archivio di Stato di Milano (ASMi), Fondi camerali p.a., 256; Archivio della Soprintendenza dei Beni Architettonici e del Paesaggio di Milano (AS BB AA PP Mi), cartella E/5/160; Archivio Borromeo Isola Bella, Chiese nei territori dalla A alla B, Arona; Biblioteca Ambrosiana di Milano (BAM), S 131 Sup., LVI-LVII e S 132 Sup., CXXIX. 
Le porte apparivano essere "piutosto fori, \& buche nelle mura, che porte convenienti a così gran Metropoli". ${ }^{5}$ Il problema di una sistemazione del principale ingresso in città era già stato posto negli anni precedenti, ma fu la programmata venuta nel 1597 di Gregoria d'Austria, figlia dell'arciduca Carlo di Stiria e promessa sposa del principe Filippo, a dare lo spunto per festeggiamenti anche con l'erezione di archi trionfali (Fig. 2). Ma la sua morte prematura bloccò il progetto fino all'anno successivo, quando il principe si fidanzò con la sorella minore di Gregoria, Margherita, e i preparativi ripresero: l'ingresso trionfale di Margherita a Milano sarebbe avvenuto durante il suo viaggio verso la Spagna per conoscere lo sposo. L'evento è celebrato anche nella moneta-medaglia custodita nel medagliere del Castello Sforzesco (Fig. 3).

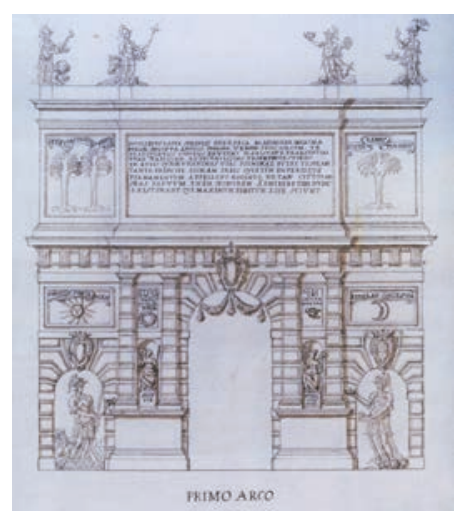

Fig. 2 - Porta Romana nel disegno inserito nel manoscritto di Guido Mazenta (?), Idea per l'arco da erigersi a Porta Romana in onore della principessa Gregoria d'Austria, 1597 (Madrid, Biblioteca Nacional, MS 2908).

Per decidere le iniziative della Città in onore dell'illustre ospite fu eletta una commissione scelta tra i membri del Consiglio Generale e i Decurioni, i quali il 28 luglio 1598 riferivano di aver stabilito che "la porta della città per la quale doverà entrare la detta Signora, che sarà

5 G. MAZENTA, Apparato fatto dalla città di Milano per ricevere la serenissima Regina D. Margarita d'Austria sposata al potentiss. Re di Spagna D. Filippo III nostro signore, Milano, nella stamperia del q. Pacifico Pontio, 1598 (seconda edizione 1599). 
Porta Romana, si faccia di vivo et materia stabile a perpetua memoria" ossia in pietra, riprendendo il disegno fatto l'anno precedente. ${ }^{6}$
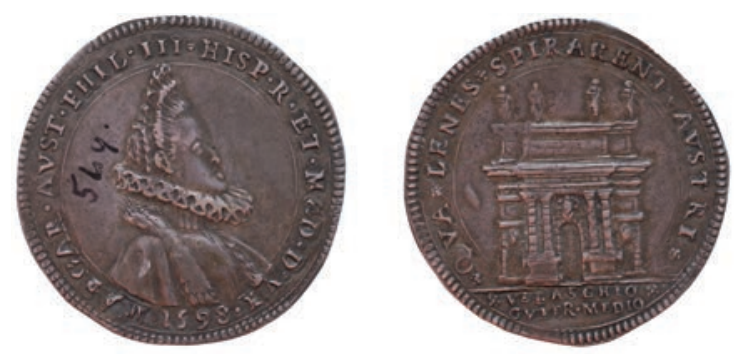

Fig. 3 - Moneta commemorativa dell'ingresso in città di Margherita d'Austria Regina di Spagna con l'effige della regina e con l'arco di Porta Romana

(Milano, Civiche Raccolte Numismatiche, Medagliere Castello Sforzesco).

Si trova anche in L. Candrini, L'Arco di Porta Romana, cit. p. 399.

Dopo essere state "pubblici passeggi" tra il Sette e l'Ottocento, le Mura non sono state comprese dai principali personaggi milanesi né reinserite nel circuito vitale urbano, forse anche perché non hanno mai svolto una reale funzione difensiva, ma solo daziaria; attribuendo loro probabilmente un significato negativo anche come limite al progresso ottocentesco e testimonianza di un periodo di dominazione straniera, di fatto ne hanno decretato la fine. ${ }^{7}$ Le demolizioni e la mancanza di cura che dal Piano Beruto (1884-88) si sono susseguiti in modo costante, per piccoli e grandi episodi, dimostrano, in generale, il mancato riconoscimento dei loro molteplici valori testimoniali di carattere storico-simbolico e architettonico-costruttivo. ${ }^{8}$ Vale la pena ricordare che Porta Romana ha rappresentato da sempre l'ingresso principale alla città di Milano, e ricordiamo che già nella cinta muraria di età comunale era

6 Relazione e fascicoli allegati del Progetto definitivo, 2006: Restauro conservativo dell'Arco di Porta Romana, da p. 3 e Documentazione iconografica.

7 Per le vicende degli ultimi due secoli si è consultato, presso gli archivi della Soprintendenza per i Beni Architettonici e per il Paesaggio, l'unica cartella presente, segnatura C/1/594 Milano- Mura Spagnole. In realtà ci sono poche testimonianze di voci in difesa delle Mura come i Soprintendenti Modigliani e Crema che però poco o nulla hanno potuto ottenere nel secolo XIX.

8 Si rimanda a M.T. FIORIO, "Opus turrium et portarum": le sculture di Porta Romana, in Milano e la Lombardia in età comunale. Secoli XI-XIII, Catalogo della mostra, Milano 1993, pp. 189-192. 
stata realizzata la struttura monumentale di più alto valore simbolico della città medievale, demolita anch'essa alla fine del Settecento. Anche l'Arco di Porta Romana risalente al 1598 ha subito nel 1907 le operazioni di isolamento dalle cortine murarie del sistema difensivo (Fig. 4) e la risistemazione complessiva dell'area nel 1955, sistemazione che ha conferito al monumento il ruolo attuale di spartitraffico (Fig. 5), causandone un incremento di degrado: il deposito superficiale, causato dal particellato atmosferico, si trasforma in deposito più compatto e spesso, fino a diventare crosta con la presenza di gesso di neoformazione e di sali a causa delle condizioni ambientali e dall'elevato livello di inquinamento, causato principalmente dal traffico automobilistico.

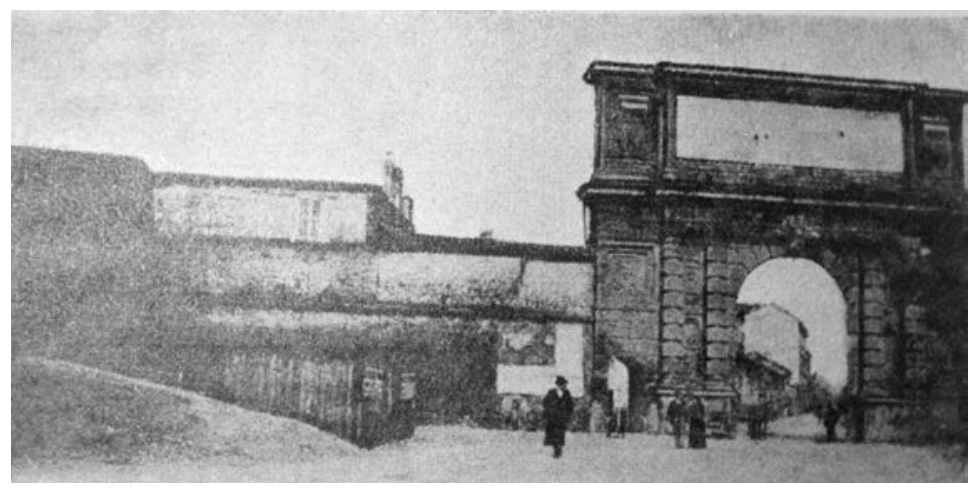

Fig. 4 - Fotografia storica di Porta Romana verso il 1900, il monumento è ancora fiancheggiato dalle Mura. Da L. Candrini, L'Arco di Porta Romana, p. 400.

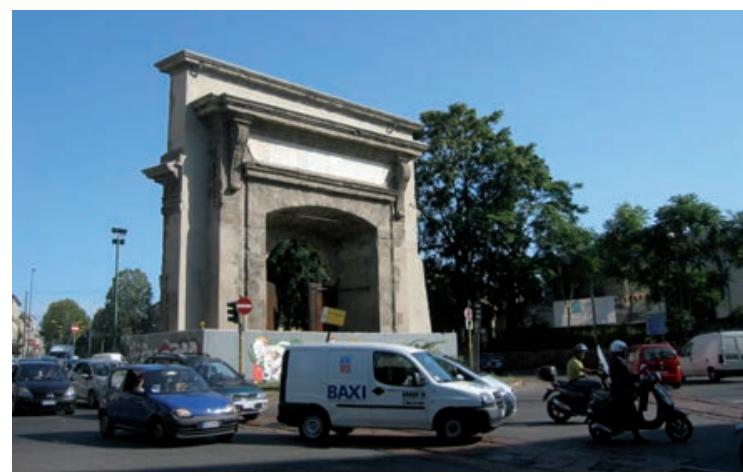

Fig. 5 - Fotografia di Porta Romana del 2009, il monumento è isolato secondo la sistemazione attuale della piazza. 
Ecco che l'intervento di conservazione delle Mura Spagnole di Milano, avvenuto tra il 2006 e il 2009, si è basato sul tentativo di cambiare tendenza: da una parte riconoscere i valori monumentali del complesso e portarli all'attenzione della comunità, dall'altro indirizzare il restauro verso le metodologie più corrette rispetto al progetto definitivo attraverso i criteri più aggiornati del processo conservativo di conoscenza, diagnostica e cura. Tale processo si è relazionato anche a un sentire tali luoghi "emozionanti" grazie al potere del frammento che evoca il tutto, del "margine" del tracciato urbano dalla cerchia delle mura ormai poco riconoscibile, della "soglia" come passaggio di un dentro e di un fuori del sistema difensivo decontestualizzato. Alla scala architettonica, come rudere, le Mura diventano documento di un saper fare, memoria di cui non si vorrebbe perdere oltre i molteplici valori testimoniali.

\section{STATO Di CONSERVAZIONE PRECEDENTE ALL'INTERVENTO}

\subsection{Localizzazione e descrizione dei tratti di mura}

Nella Fig. 6 sono identificati in rosso i tratti delle Mura oggetto dell'intervento eseguito tra il 2006 e il 2009, compresi in una porzione posta circa a sud-est del sistema fortificato cinquecentesco (attuale circonvallazione esterna):

Tratto T1 cavaliere tra viale Caldara e viale Monte Nero;

Tratto T2 bastione di Porta Romana, circoscritto tra piazza Medaglie D'oro, viale Filippetti e viale Sabotino, e adiacente tratto di mura;

Tratto T3 mura bastionate con cavaliere compreso tra viale Filippetti e viale Sabotino;

Tratto T4 cavaliere tra viale Beatrice d'Este e via Patellani;

Tratto T5 punto di difesa avanzata corrispondente a viale Beatrice d'Este e largo Isabella d'Aragona;

Tratto T6 punto di difesa avanzata e tratto di mura comprese tra viale Beatrice d'Este fino a porta Ludovica.

Il primo tratto T1 corrisponde al cavaliere di viale Caldara-viale Monte Nero, presenta l'impianto di "giardino pubblico con memoria monumentale affiorante" con scale a rocaille e parterre a ghiaia, delimitato da un parapetto in ferro e tratti di Mura. Completano l'arredo urbano la creazione di aree di sosta e terrazzamenti. 
Il secondo tratto T2, comprende il bastione di Porta Romana, unico bastione cittadino conservato. Si caratterizza per un rivestimento basamentale in ceppo d'Adda, un paramento esterno con tratto a scar$\mathrm{pa}$, separato dal tratto superiore di muratura ad andamento verticale mediante un cordolo marcapiano a toro sempre in ceppo; all'epoca del restauro era concluso da cordolature cementizie e copertina sommitale risalente ad anni recenti. Verso l'interno del bastione è possibile trovare l'unico esempio di barbacani e dei relativi archi di scarico, originariamente protetti da terrapieni.

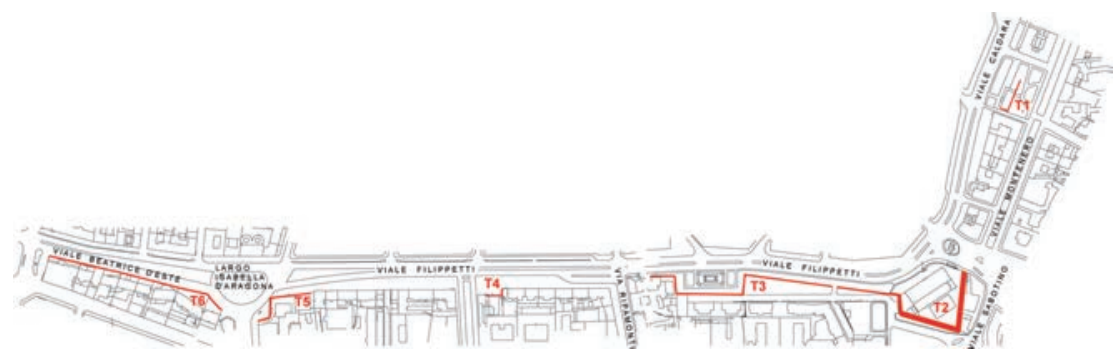

Fig. 6-Schema dei lotti di intervento: tratti T1-T6.

Il terzo tratto di viale Filippetti T3 è connotato dall'intervento degli anni Sessanta, attuazione del progetto del 1953, che ha trasformato l'area corrispondente al cavaliere in giardino pubblico. Le parti interne della muratura risultano essere state interpretate come quinte scenografiche poste a sfondo della fontana.

Anche i resti delle mura presenti lungo viale Filippetti T4, largo Isabella d'Aragona T5, viale Beatrice D'Este fino a Porta Ludovica T6, sono il risultato delle trasformazioni del ventennio compreso tra il 1950 e il 1970; in particolare, le varianti ai Piani particolareggiati del 1953 e le molte autorizzazioni, anche da parte della Soprintendenza, a parziali demolizioni di tratti, persino nell'ordine degli 80 metri lineari. È evidente che le Mura erano viste come semplici cinte di delimitazione per le proprietà private in costruzione, non riconoscendone il loro valore monumentale.

\subsection{Materiali e fenomeni di degrado}

Con la consulenza del dott. Marco Realini e in particolare del 
dott. Alberto Villa9 si è preso in considerazione l'interrelazione tra il manufatto e le specie arboree.

I materiali che costituiscono la muratura, malte e mattoni, rappresentano infatti un substrato favorevole allo sviluppo della vegetazione per l'elevata porosità presente, che favorisce sia la ritenzione di umidità sia la penetrazione meccanica delle radici, anche per il fatto che i giunti di malta e i vari interstizi presenti nelle murature sono luoghi privilegiati per il deposito di polveri, materiali organici, ecc.. La compresenza di adeguata illuminazione che permette l'attività fotosintetica, di elevato tenore di umidità del substrato causato dalla pioggia, dall'umidità di risalita, da percolazione o condensa, e le condizioni ambientali favorevoli, hanno portato a una crescita biologica originata da comunità sostanzialmente erbacee, ma giunta fino a comunità arbustive e arboree più complesse e aggressive rispetto al substrato. Ricordiamo che, la crescita biologica è favorita da quei processi di degrado che portano all'aumento di porosità, al decoesionamento e alla fratturazione dei materiali esistenti, aumentando la "bioricettività" ${ }^{10}$ del substrato; inoltre, le specie arboree risultano essere in grado di vivere in ambienti con un regime idrico discontinuo e poveri dal punto di vista nutritivo.

L'esecuzione delle operazioni di controllo degli agenti del degrado a prima vista potrebbero risultare relativamente semplici, invece sono alquanto complesse poiché esistono da un lato la resistenza allo strappo delle radici, che potrebbero ricacciare se dovessero restare vive in sito, e dall'altro l'impossibilità di raggiungere con mezzi meccanici le radici penetrate nelle strutture senza recare danni ai manufatti.

I tratti di mura presentavano emergenze decisamente differenziate in relazione alle vegetazione spontanea: a rischio di crollo, in parte già avvenuto (Fig. 7), fratturati con disgregazione delle malte e distacchi di intere parti del paramento murario in mattoni. Lo sviluppo vegetale

9 Esperto di Beni Culturali e membro della Commissione Normal; ha collaborato alla realizzazione di molti progetti nazionali per la protezione di aree archeologiche dalla vegetazione infestante. Colgo l'occasione per ringraziarlo della disponibilità e ricchezza di dati e testimonianze personali che mi ha trasferito durante le nostre passeggiate (circa $2 \mathrm{~km}$ di tratti), nelle visite di sopralluogo al cantiere.

10 CANEVA G., "La colonizzazione biologica della cinta muraria di Lucca: linee guida di un progetto di studio di biodeterioramento e di biomonitoraggio", in Atti del convegno Le Mura di Lucca, Lucca 17-19 maggio 2001, GIUSTI M.A. (a cura di), Alinea, Firenze, 2005, p. 194. 
"non ostacolato" ha portato al collasso strutturale dei manufatti, determinando il loro "ruinare", ${ }_{11}^{11}$ soprattutto nei tratti T2 e T3 (Fig. 8).

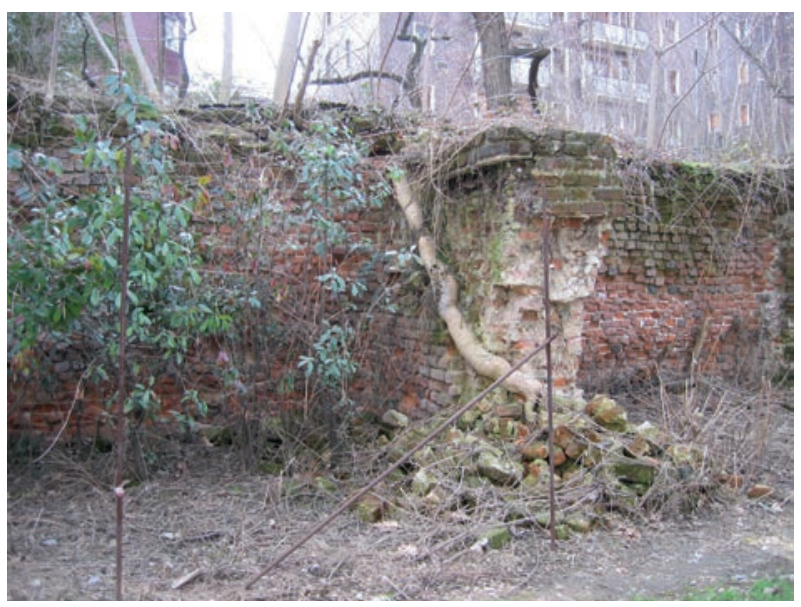

Fig. 7 - Fotografia del 2006, barbacane del tratto T2 di viale Filippetti; in particolare è evidente il danno prodotto da una grossa radice.

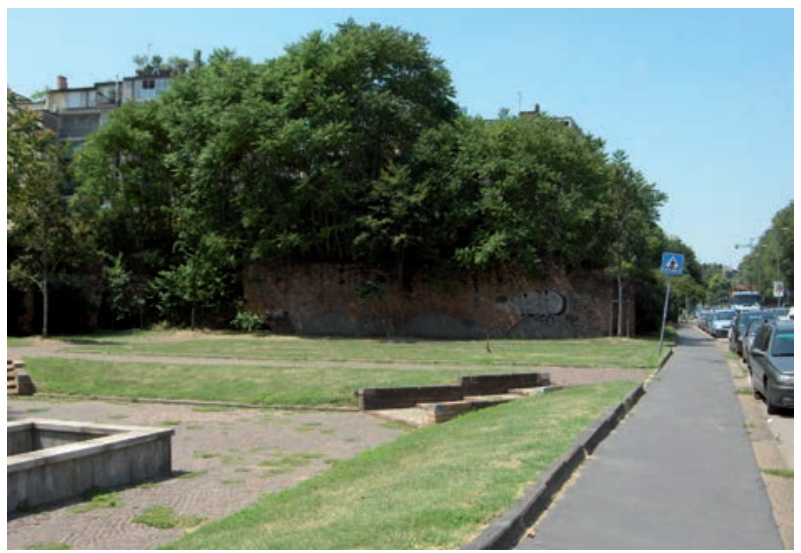

Fig. 8 - Fotografia del 2006, fianco del cavaliere del tratto T3 di viale Filippetti; è visibile la crescita incontrollata di arbusti di grandi dimensioni, alloggiati in particolar modo all'interno del fianco, che si comporta come una "vasca" di contenimento.

11 CANEVA G., op. cit., 2005, p. 195. 
A partire dal progetto definitivo, si è proceduto a una serie di sopralluoghi con l'obiettivo di verificare le entità vegetali che crescono sui paramenti, maggiormente responsabili delle azioni di degrado, e nelle loro immediate vicinanze. A seconda del tratto analizzato, le specie individuate apparivano in molti casi uniformemente diffuse mentre altre erano più localizzate.

Tra le specie distribuite con una buona frequenza ovunque sulle mura c'erano: Ailanto, Bagolaro, Sedum, Vite americana, Edera e Parietaria. Queste ultime due si trovavano per parti di murature molto estese, lungo ogni tratto, particolarmente presenti sulle cortine orientate a nord e sud (prive di illuminazione e umide perché protette da vegetazione); nel tratto T6 Edera e Vite americana si presentavano folte e rigogliose su entrambi i lati delle mura, denotando una scelta estetica da parte dei privati che le avevano piantate. Particolarmente preoccupante per quanto riguarda la sua diffusione e collocazione era l'Ailanto: introdotto dalla Cina in Europa verso la fine del XVIII secolo, ha trovato in ambiente mediterraneo un perfetto acclimatamento, diventando una specie infestante di difficile controllo sia per la rapidità di sviluppo vegetativo che per i danni determinati dal suo insediamento sulle strutture murarie.

Si sottolinea che pur essendo il termine di specie infestante riferito a un concetto variabile e soggettivo, di norma lo si utilizza in quei casi in cui lo sviluppo di una specie interferisce con le attività umane o rappresenta un elemento di squilibrio nella naturale dinamica degli ecosistemi. ${ }^{12}$

Per quanto riguarda la vegetazione circostante le Mura, le piante e gli arbusti presenti costituivano una popolazione caotica e anomala per la compresenza di specie piantate con intento ornamentale, ad esempio gli Allori tra i barbacani dei tratti T2 e T3 o, arbitrariamente, da parte di cittadini (Aucuba, piccolo Abete, ecc..), e altre cresciute per semina spontanea, soprattutto Ailanti. L'assenza di uno studio agronomico delle specie idonee all'ambiente esistente ha creato casi di sofferenza e attacchi virali per le quali si è previsto l'abbattimento ${ }^{13}$, di concerto con gli enti di tutela: Forestale e Soprintendenza.

12 CANEVA G., "Il problema della crescita di Ailanthus altissima (Miller) Swingle nelle zone archeologiche e monumentali" in Atti del Convegno Le Pietre nell'Architettura: Struttura e Superfici, Bressanone, 25-28 Giugno, 1991, pagg. 225-233.

13 La classificazione e ulteriori dettagli sulla vegetazione si può trovare nel fascicolo Allegati alla Relazione del Progetto esecutivo, 2006. 


\subsection{Stato di conservazione della muratura}

Si è visto come il degrado causato dagli organismi biologici si articola attraverso i meccanismi fisici di decoesione e disgregazione del substrato effettuati dalla spinta e dalla penetrazione di strutture vitali nel materiale, che portano a danni di natura meccanica o da alterazione di tipo chimico.

La scelta di liberare dai terrapieni e lasciare a vista la maggior parte dei muri originariamente costruiti per essere ricoperti di terra, ha accelerato il processo di degrado perché parti del manufatto, non costruite per essere esposte agli agenti atmosferici, presentano una porosità maggiore rispetto a quella di un paramento esterno, realizzato con funzione di protezione del nucleo. Inoltre, non prevedere per tutto lo sviluppo una protezione superiore e l'assenza di una progettazione delle aree a verde e del controllo della crescita della vegetazione, ha determinato le situazioni di dissesti, crollo e perdita di consistenti porzioni di materia già denunciate.

Tra le principali cause di degrado delle murature troviamo l'acqua, sia allo stato liquido che a quello di vapore e, quindi, condensa. Esistono differenti vie di ingresso dell'acqua all'interno delle strutture murarie: essendo i mattoni e le malte materiali porosi sono permeabili all'acqua stessa. In molti casi, soprattutto sulla cortina a scarpa, i giunti di malta erano talmente degradati da risultare fortemente arretrati rispetto alla superficie, favorendo la penetrazione dell'acqua meteorica. Un'altra via privilegiata è la risalita dal terreno per capillarità oppure la discesa dall'alto per gravità, soprattutto in corrispondenza delle fessure e delle fratture createsi per la presenza della vegetazione e della mancata manutenzione.

Dalle analisi termografiche all'infrarosso e dai test gravimetrici, effettuati sia sul bastione che in un tratto di viale Filippetti, è risultato che le infiltrazioni erano presenti nella parte sommitale, tra il paramento e il riempimento della muratura e tra le mura e i contrafforti. La distribuzione dell'acqua all'interno delle strutture era presente con una maggiore concentrazione alla base delle murature, nei punti in cui si generavano dei piani interni di scorrimento che permettevano alle infiltrazioni di diffondersi rapidamente per gravità.

I processi degenerativi o degradativi di natura chimico-fisica sono accelerati dalle particolari condizioni climatiche di Milano, determinate in gran parte dalla conformazione orografica dell'area, essendo la pianura 
padana circondata su tre lati da catene montuose che si estendono fino a quote elevate, determinando così peculiarità climatologiche sia dal punto di vista fisico che da quello dinamico, influenzando in modo determinante le capacità dispersive dell'atmosfera e quindi le condizioni di accumulo degli inquinanti, soprattutto nel periodo invernale.

Per la molteplicità delle situazioni riscontrate rispetto alle tessiture murarie, il degrado degli elementi, variabile per intensità da zona a zona, era presente sia nei giunti sia sugli strati più superficiali dei mattoni, particolarmente su quelli meno cotti o nei casi in cui erano presenti stilature cementizie. La muratura si presentava con caratteristiche differenti a seconda che fosse conservato il paramento a vista, a tessitura regolare, con corsi allineati e malta di allettamento di spessore uniforme, oppure che fossero esposte parti più interne. In questi casi già presente sopra i mattoni non erano più complanari, disposti senza corsi preferenziali e con la malta di allettamento stesa in modo disomogeneo. Lo stato delle mura era però anche il risultato di una lunga assenza di manutenzione che ha contribuito ad accelerare il degrado dell'apparato murario fino ai casi di dissesto e di perdita di ampie porzioni del costruito.

Proprio per la stretta relazione tra la patologia di degrado e il substrato interessato si è proceduto alla schedatura degli elementi e dei loro materiali, dei fenomeni di degrado che descrivono ed esemplificano quanto riscontrato rispetto al materiale specifico, al rilevamento del quadro fessurativo e del dissesto (analisi mancanti nel progetto definitivo).

Ricordiamo che se la superficie dell'opera è il luogo di tutte le trasformazioni fisiche e chimiche prodotte dall'interazione tra l'ambiente e il materiale, essa è anche il luogo della percezione estetica e la superficie da conservare in termini materici.

\section{INTERVENTO}

\subsection{Premessa metodologica}

Il progetto definitivo è stato considerato alla luce dell'analisi dello stato di fatto del manufatto, definendo con molta attenzione eventuali rischi, controindicazioni o effetti collaterali dannosi. Nei casi di incertezza dei risultati alla prova del tempo, alla luce della complessità della reale condizione riscontrata, si è optato per una soluzione di "non intervento" in quanto è parsa la scelta più conservativa. Infatti, ogni inter- 
vento di restauro deve essere valutato con la necessaria prudenza, evitando i rischi derivanti da una teorica fiducia nella tecnica, illudendosi che si possa sempre risolvere una problematica complessa con un singolo intervento "miracoloso". Ovviamente, il progetto esecutivo e l'intervento realizzato sono stati la conseguenza di un determinato approccio metodologico e la scelta di principi guida definiti: l'istanza dell'intervento è stata la conservazione dei materiali presenti ossia la salvaguardia della durata fisica dell'opera e, quindi, delle tracce dell'uomo e anche degli eventuali mutamenti causati dal trascorrere del tempo, per il periodo che sia il più lungo possibile.

\subsection{Lavorazioni}

L'intervento si è articolato in operazioni preliminari, sulla vegetazione, puliture, stuccature e sigillature, consolidamenti, opere di protezione, opere complementari. All'interno di queste categorie i singoli interventi sono stati relazionati ai differenti materiali presenti nel monumento, nonché alle relative patologie di degrado. Tutte le operazioni di restauro sono state eseguite da strutture operative con Sistema di Qualità certificato, dotato di SOA per la categoria di lavorazione OG2 e OS2. ${ }^{14}$

Di seguito riportiamo le lavorazioni eseguite. ${ }^{15}$

Le operazioni preliminari hanno compreso: l'eliminazione degli arbusti situati all'interno delle aree comprese tra i barbacani o adiacenti al manufatto, in modo da liberare le mura e le zone limitrofe, consentendo l'allestimento del cantiere e l'esecuzione delle operazioni di restauro e consolidamento del manufatto; il recupero dei mattoni, loro accatastamento su bancali nelle immediate vicinanze, pulitura e successivo loro reimpiego nelle lavorazioni di consolidamento e piccole integrazioni; la messa in sicurezza di porzioni di muratura pericolanti, nei casi di rischio di crollo o di forte distacco di parti di muratura in mattoni o di singoli elementi (mattoni o pietra), mediante opere provvisionali quali puntelli

14 Si tratta delle categorie che si possono ottenere con l'attestazione SOA: OG2 Restauro e manutenzione dei beni immobili sottoposti a tutela e OS2 Superfici decorate di beni immobili del patrimonio culturale e beni culturali mobili di interesse storico, artistico, archeologico ed etnoantropologico

15 Il presente paragrafo riprende ampiamente quanto già riportato nel Consuntivo scientifico, redatto nel 2009, al termine dei lavori, a cura della sottoscritta e dell'Arch. Lorena Bauce. 
(Fig. 9), legature e stuccature provvisorie, a garanzia della stabilità per consentire le operazioni successive; il preconsolidamento delle superfici lapidee particolarmente degradate con applicazione di prodotto consolidante (esteri dell'acido silicico), applicato a pennello, al fine di consentire le successive operazioni di pulitura senza perdita di materiale.
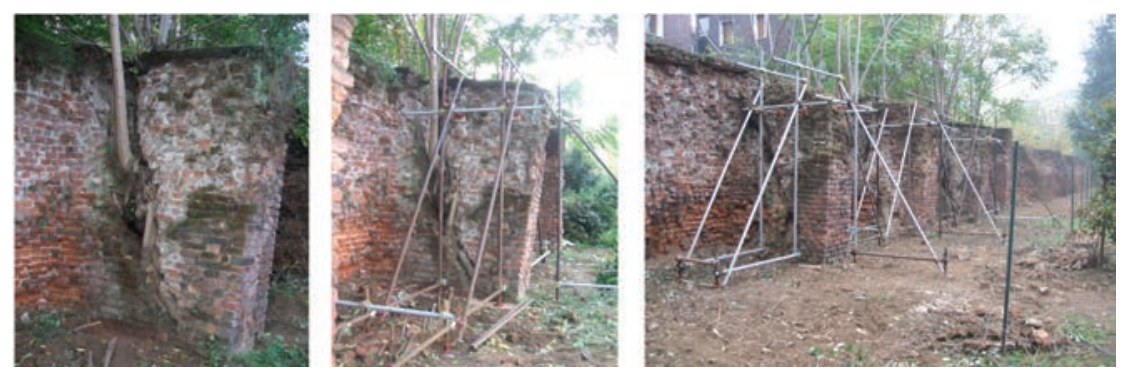

Fig. 9 - Sequenza fotografica della messa in sicurezza dei barbacani a rischio di crollo, tratto T3 corrispondente al cavaliere di viale Filippetti.

Per quanto riguarda la vegetazione e l'attacco biologico, si ricorda che i principali biodeteriogeni sono parte di un vasto ambito che comprende batteri (autotrofi ed eterotrofi), attinomiceti, funghi, cianobatteri e alghe (cianoficee e cloroficee), licheni (crostosi, fogliosi e fruticosi), briofite (muschi ed epatiche), piante superiori (erbacee, arbustive e arboree) e animali (insetti, lumache e uccelli); per ogni categoria di biodeteriogeni esistono appropriati metodi di controllo, che possono essere di tipo meccanico, fisico e chimico selettivi.

Nel caso specifico si è proceduto richiamando trattamenti che vengono eseguiti in ambito archeologico e monumentale: metodi meccanici, attentamente integrati con un misurato uso di quelli chimici. In particolare si ricorda che esiste una categoria di specie, detta "ruderale" che alligna in preferenza proprio sul costruito. Tali specie ruderali si nutrono a spese dei materiali da costruzioni quali le malte, gli elementi lapidei artificiali e anche naturali. Da tutto ciò è emersa la necessità di definire i migliori metodi di asportazione per le specie da eliminare e quelle che potevano sopravvivere accanto alle mura, fino alla questione più grave che ha riguardato i casi degli Ailanti i cui tronchi si sono intersecati con l'apparecchio murario.

Le operazioni di restauro sulle strutture murarie, in laterizi a vista, hanno previsto trattamenti preliminari per l'eliminazione sia della vegetazione erbacea che di quella arbustiva e ad alto fusto. 
Il primo intervento ha previsto il taglio dei rami e la rimozione delle masse non aderenti alla muratura; successivamente si è proceduto all'eliminazione della vegetazione ruderale e infestante mediante l'applicazione di prodotti chimici sistemici. Per quanto riguarda patina biologica, funghi, batteri, muffe, alghe e muschio si è proceduto su tutte le superfici interessate con un trattamento a spruzzo; un secondo trattamento è stato eseguito con un erbicida sistemico liquido solubile, a base di glifosate, ${ }^{16}$ scelto per la bassa tossicità e assenza di attività residuale. Il prodotto agisce su sedum, parietaria, edera, vite americana, per assorbimento da parte delle foglie e viene traslocato per via sistemica sino alle radici e agli organi perennanti (Fig. 10).
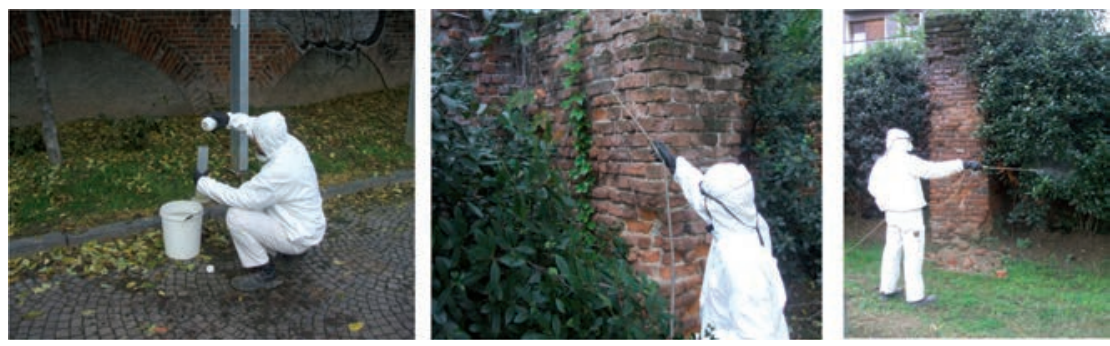

Fig. 10 - Sequenza fotografica del trattamento del verde con glifosate (erbicida sistemico).

Dopo un tempo di attesa di circa 3 settimane si è passati alla rimozione della vegetazione umifera ed erbacea "morta" per l'azione del biocida.

Come già evidenziato nel testo, per motivi di maggiore efficacia e minor rischio di lesioni al substrato, si è preferito un trattamento chimico rispetto al solo intervento meccanico in quanto la semplice asportazione meccanica non risulta efficace, a meno di non poter rimuovere in maniera completa gli apparati radicali, e inoltre l'asportazione diretta di strutture vegetanti con radici significative e profonde può ledere il substrato a causa della resistenza delle radici stesse. Invece, dopo l'ap-

16 Per il Glyphosate è stato condotto uno studio in occasione del restauro delle Mura di Ferrara. Cfr. PASETTI A., MASSA V., COZZI E., "Studio dell'impatto del 'diserbo chimico' su paramenti in laterizio a vista. Il caso delle Mura di Ferrara" in Atti del Convegno Le Superfici dell'Architettura: il cotto. Caratterizzazioni e trattamenti, Bressanone, 30/6 - 3/7, 1992, pagg. 743-747. 
plicazione di uno specifico biocida, radici e capillizio subiscono una notevole riduzione di volume dovuta alla totale perdita di acqua, consentendo di poter disalveolare, senza danni ai manufatti, il materiale vegetale che vi si era insinuato.

Per quanto riguarda le piante che avevano sconnesso le strutture murarie, compenetrandole profondamente, e che non consentivano la loro estirpazione senza provocare danni alla muratura stessa, si è proceduto con il taglio della pianta, lasciandone temporaneamente parte in situ, e applicando il successivo trattatamento di "devitalizzazione". Tale procedimento è stato eseguito mediante iniezioni di diserbante liquido (principio attivo Picloram), previa realizzazione di opportuni fori, eseguiti con trapano, al fine di raggiungere i vasi conduttori (trachee) delle infestanti arbustive e legnose (Fig. 11).
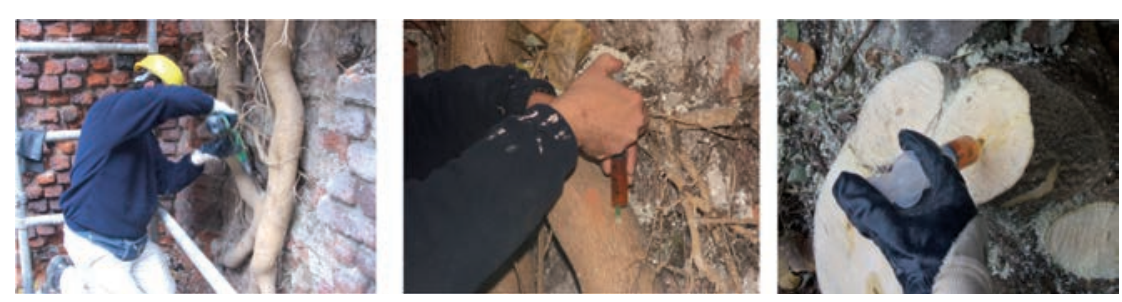

Fig. 11 - Sequenza fotografica del trattamento di "devitalizzazione" delle radici con le iniezioni di Picloram.

Dopo la totale "devitalizzazione" del legno (con un tempo variabile in funzione della stagione e della risposta dell'elemento vegetale), si è proceduto in due differenti modi: mediante rimozione completa del tronco, ove era possibile, attraverso lo smontaggio di piccole porzioni di muratura e tagli successivi; con la "mummificazione" della pianta ossia la sua conservazione in situ quando, invece, l'eliminazione della massa vegetale avrebbe comportato la perdita di elevate parti di muratura. Questa seconda tecnica prevede l'annegamento della massa vegetale all'interno della malta o materiali da costruzione, per privare di ossigeno e di luce l'apparato radicale "morto", al fine di evitare il degrado dello stesso (marcescenza). Il procedimento è visibile anche nell'ultima fotografia della sequenza illustrata nella Fig. 12.

A seguire sono state eseguite le puliture utilizzando i criteri di selettività, gradualità e controllabilità dei diversi metodi, e rispettando la superficie del materiale, senza produrre discontinuità superficiali o provocare la 
formazione di sottoprodotti dannosi per la conservazione del materiale lapideo. L'intervento sulla muratura (malta, mattoni e pietra) ha previsto una pulitura ad acqua diffusa e generalizzata, mediante idropulitrice erogante acqua deionizzata, integrata con spazzolatura (spazzole morbide) e AB57 nelle zone con depositi più resistenti. L'impiego successivo e puntuale di microaeroabrasivatrice di precisione è stato utilizzato nei casi di incrostazioni e depositi tenaci, non rimossi con la pulitura ad acqua e AB57. L'inerte utilizzato è stato il carbonato di calcio, mentre la pressione di impatto è stata molto bassa e variabile a seconda delle zone. Si fa presente che per la forte adesione del deposito ai mattoni compatti, non è stato possibile ovunque rimuovere completamente la crosta nera, pena la perdita degli strati superficiali del mattone.
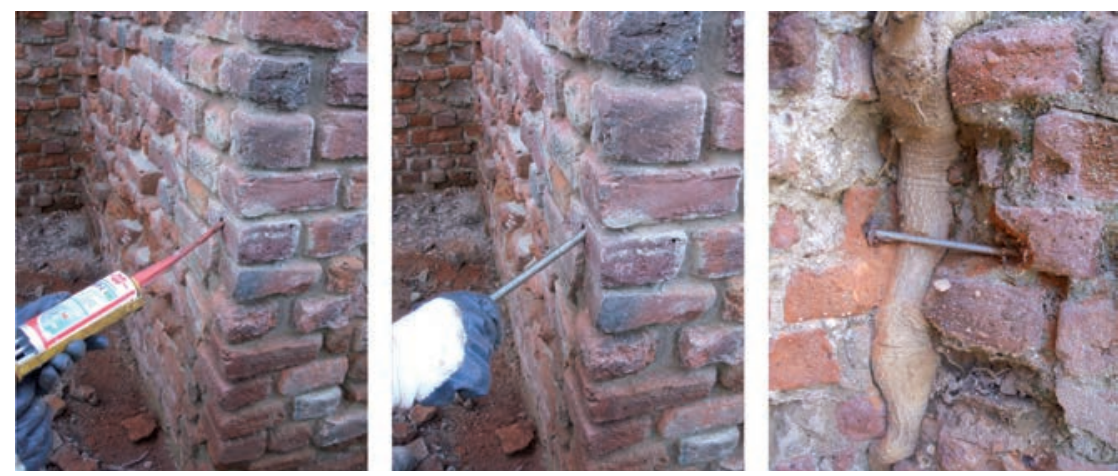

Fig. 12 - Sequenza fotografica della fase di consolidamento mediante barre in acciaio inox filettate. Si può notare nella terza foto la fase di "mummificazione" della radice.

Impacchi di polpa di cellulosa a fibra media di 200 micron e acqua distillata sono stati eseguiti per l'estrazione di sali, mentre per la rimozione delle scritte vandaliche si è proceduto con una prima applicazione di prodotto a base di cloruro di metilene, un lavaggio successivo e un ritocco con la microaeroabrasivatrice. Inoltre, per quanto riguarda l'abbassamento delle macchie di ossidi di ferro si sono effettuati impacchi a base di EDTA bisodico, usando sempre la polpa di carta come ispessente.

Nella scelta progettuale le stuccature e le sigillature hanno rivestito un ruolo fondamentale per la protezione delle mura, soprattutto in riferimento al fenomeno della semina ambientale ossia la capacità di diffondere da parte delle piante i propri semi anche lontano dal luogo di emissione e 
la capacità di questi ultimi di attendere le condizioni ottimali per germinare. Essendo il muro interessato dalla presenza di umidità, prevalentemente di natura discendente, il tipo di malta del giunto ha assunto un'importanza notevole sia per quanto riguarda la distribuzione dell'acqua all'interno del muro sia per quanto riguarda l'evaporazione di quest'ultima e quindi del degrado. Il fenomeno infatti è strettamente connesso anche alla velocità del flusso evaporativo che in condizioni termoigrometriche esterne costanti dipende dal tipo di porosità e soprattutto dal coefficiente di assorbimento capillare. Il criterio utilizzato per la scelta della malta del restauro si è basato principalmente sulla compatibilità chimico-fisica e meccanica rispetto all'esistente, sia nel senso dell'eventuale interferenza tra il materiale aggiunto e quello esistente sia nel senso dell'affinità di comportamento al variare delle condizioni esterne (igroscopicità, modulo elastico, deformabilità termica). Soddisfatta la compatibilità, sono stati privilegiati i criteri di durabilità, manutenibilità, formale e cromatico, orientandosi verso una riconoscibilità delle nuove malte, ottenuta però evitando violente intromissioni nella percezione dell'immagine dell'opera nella sua integrità e in relazione all'ambiente circostante. Per questo motivo si è optato per una differenziazione delle nuove malte formulate con calce idraulica naturale, polvere di marmo nera, gialla e rossa, graniglia e/o sabbia vagliata, a seconda del materiale di supporto. Stuccature delle fessurazioni, risarciture e stilature delle malte di allettamento dei giunti sono state precedute dalla rimozione di quelle malte cementizie o degradate (Fig. 13), dannose alla muratura o non più idonee, probabilmente eseguite in occasione di precedenti interventi, effettuata con microscalpelli.
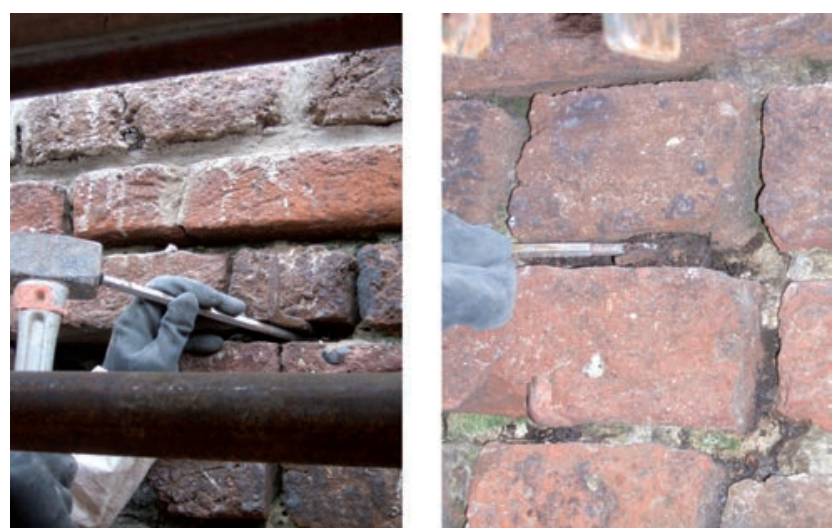

Fig. 13 - Fase di rimozione delle malte di allettamento e di stilatura non più idonee. 
L'applicazione delle nuove malte, eseguita per strati successivi stesi a mano o con la fugatrice nei tratti regolari della cortina muraria, è stata eseguita con spatolini metallici per consentire una stilatura puntuale e molto accurata. Dopo un periodo di tempo che ha consentito un primo indurimento dello strato finale, si è proceduto con la lavatura e tamponatura delle superfici con spugne di mare e acqua deionizzata al fine di porre in risalto l'aggregato, la sua dimensione e la sua specifica cromia (Fig. 14). La lavorazione si è prefissa anche lo scopo di conservare e rendere leggibile i segni, per così dire "calligrafici", che si riscontrano osservando con attenzione le diverse stilature e rifiniture dei giunti che, allo stato attuale delle conoscenze, sono l'unica testimonianza materiale delle trasformazioni o manutenzioni più o meno recenti.
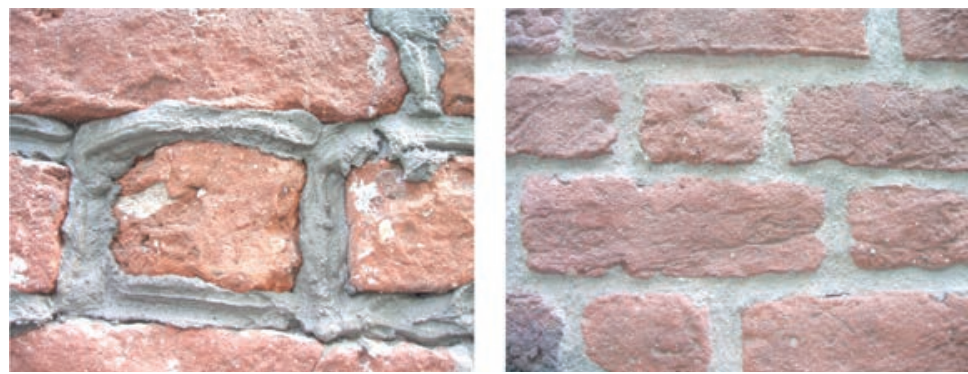

Fig. 14 - Fase di rifacimento delle malte di stilatura:

malta applicata con fugatrice (a sinistra) e malta a lavorazione conclusa.

Per le stuccature e i riempimenti delle fessurazioni profonde si è proceduto mediante colatura o iniezione in profondità di un impasto $\mathrm{a}$ base di calce idraulica naturale e sabbie vagliate (Figg. 15, 16).

Anche sui fenomeni di alveolizzazione si è operato restando sottotono, dal punto di vista cromatico, con una formulazione a base di grassello di calce, cocciopesto, polvere di marmo nera e rossa, terre coloranti.

Il consolidamento e la sigillatura delle fessurazioni profonde e dei distacchi del rivestimento in ceppo sono stati eseguiti con un impasto a base di calce idraulica naturale, polvere di marmo e sabbia vagliata. Inoltre il consolidamento profondo della muratura si è articolato attraverso due differenti procedure: con riempimenti di malta e riadesioni e con la tecnica del cuci-scuci nei casi di paramento decoeso, ammalorato o friabile. Per quanto riguarda il cuci-scuci si è proceduto con la rimozione degli elementi esterni fortemente degradati o sconnessi, la rimozione dei 
giunti di allettamento inconsistenti, la successiva pulitura delle superfici, la preparazione delle stesse, il riutilizzo o l'integrazione con mattoni di recupero. I nuovi elementi sono stati fatti fare a mano, realizzati secondo un campione prelevato dalle mura, riconoscibili per fattura, ma simili all'esistente per dimensione, colore e composizione.
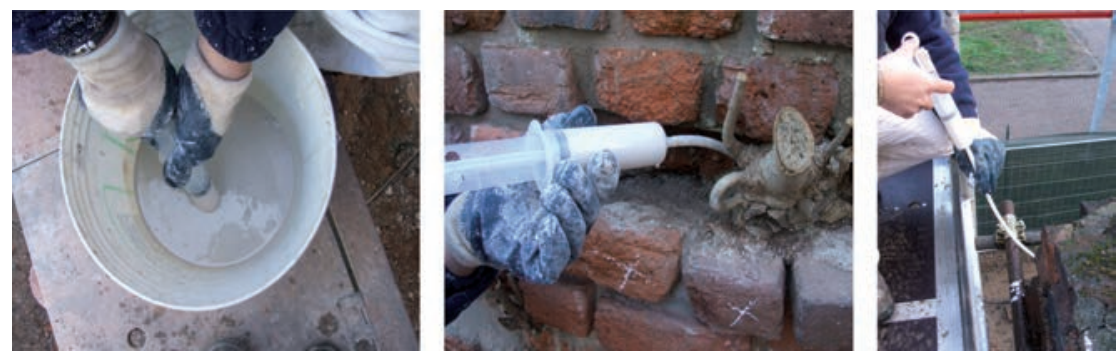

Fig. 15 - Sequenza fotografica della fase di consolidamento mediante iniezioni della muratura.
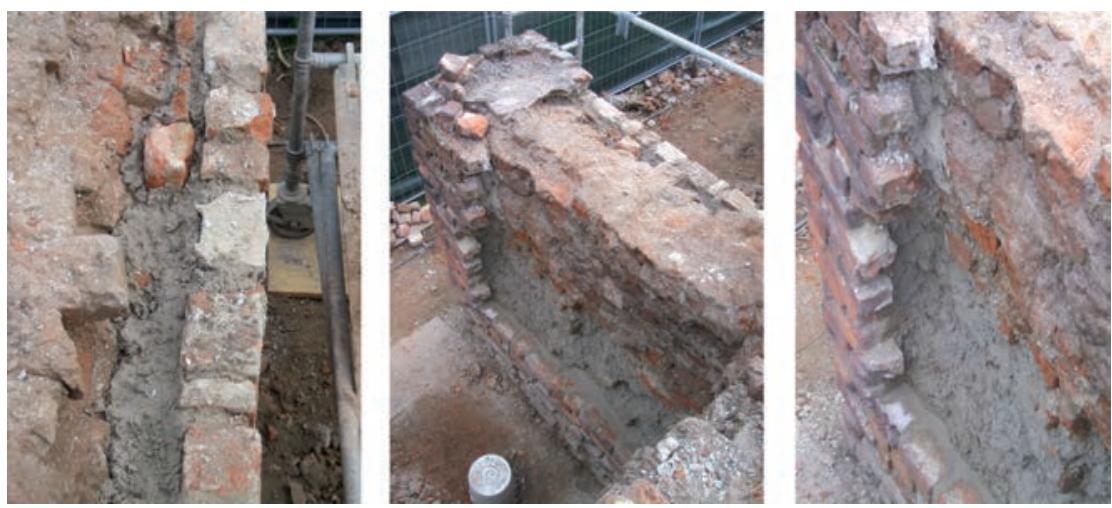

Fig. 16 - Dettagli fotografici della fase di consolidamento della muratura.

A seguito della verifica puntuale dello stato di consistenza della muratura, messo in luce dopo l'intervento sulla vegetazione, si sono inserite barre in acciaio inox filettate (Fig. 12) nei casi in cui si sono riscontrati paramenti esterni dei barbacani, probabilmente costruiti in epoca successiva rispetto alla parte centrale. Tali rivestimenti non ammorsati, ma solo accostati, hanno favorito l'azione degli apparati radicali che risalendo hanno distaccato il paramento stesso.

L'inserimento di elementi metallici è stato eseguito anche su quattro 
barbacani situati nell'angolo sinistro del cavaliere verso via Filippetti; questi barbacani si caratterizzavano per la presenza di radici e tronchi d'albero di dimensioni notevoli, che hanno distaccato la porzione mediante fessurazioni profonde, diventate le sedi dei tronchi stessi, con fenomeni di inclinazione e fuori piombo, e dissesti localizzati. Poiché si trattava di porzioni di muratura relativamente compatta e resistente, si è potuto procedere alla ricostituzione muraria del paramento mediante "cuci-scuci" e interventi di riempimento delle fessurazioni profonde e dei distacchi, aggiungendo tirantature metalliche laterali contro il ribaltamento delle porzioni ricucite (Fig. 17), anche nei casi di spinta accidentale (ad esempio per atto vandalico o urto di un mezzo di trasporto).
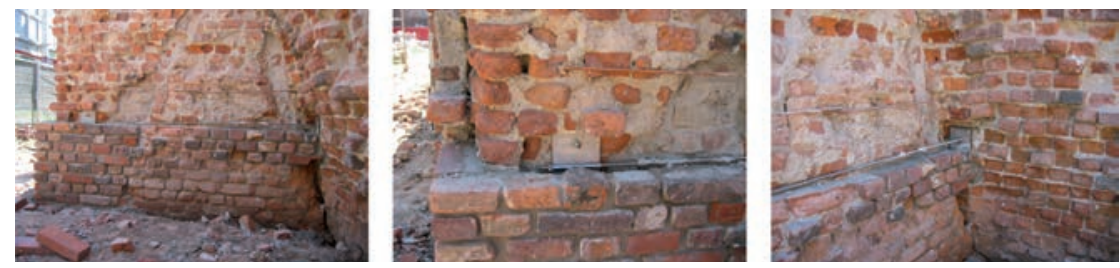

Fig. 17 - Sequenza fotografica del consolidamento dei barbacani con tiranti in acciaio inox.

In generale, l'intervento non ha previsto l'applicazione di un protettivo sull'intero paramento murario per facilitare l'evaporazione dell'acqua dalla muratura, ma il trattamento si è reso necessario puntualmente ove l'azione della pioggia di rimbalzo era più frequente.

Particolare attenzione si è posta nell'individuare differenti tipologie di copertine per la protezione del manufatto da infiltrazioni, ruscellamenti e depositi d'acqua, ad esempio la protezione della parte inferiore delle buche pontaie e dei mattoni in aggetto con realizzazione di copertina in malta, modellata per raccordare e agevolare l'allontanamento delle acque meteoriche, con caratteristiche simili a quella utilizzata nelle parti circostanti per le stilature e sigillature (calce idraulica naturale, sabbia vagliata e polvere di marmo); la copertina della parte sommitale realizzata in malta con andamento a dorso d'asino o in pendenza, stesa in due strati su supporto preventivamente pulito e preparato: lo strato di fondo è costituito da calce idraulica naturale e sabbia vagliata, mentre l'impasto della malta di finitura è stato realizzato con calce idraulica naturale, cocciopesto e polvere di marmo giallo oro e nera (Fig. 18). Le copertine hanno avuto anche lo scopo di colmare le discontinuità e le piccole mancanze presenti sulle superfici, in modo da ridurre la possibilità di infiltrazioni e ristagni. 

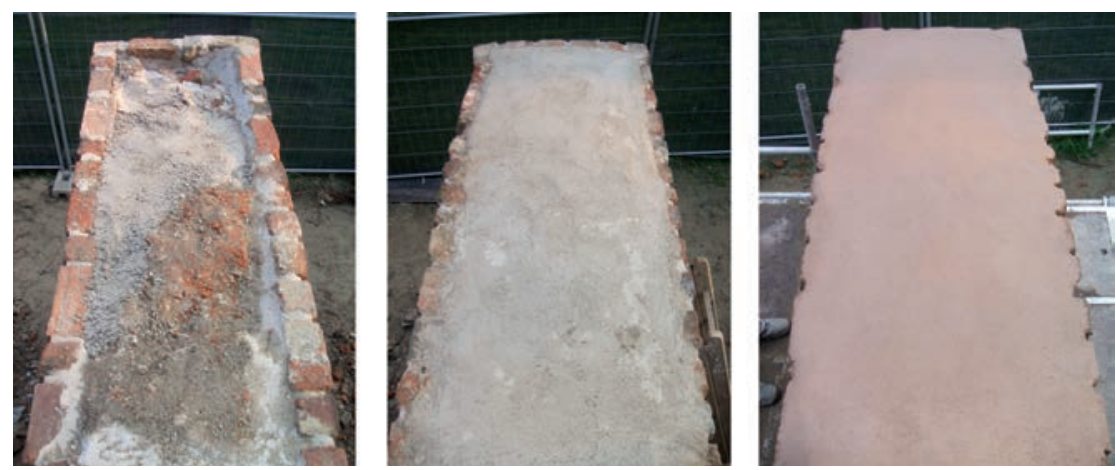

Fig. 18 - Sequenza fotografica dei vari strati della copertina sommitale posta sulle creste delle mura.

A chiusura delle buche pontaie è stato inserito un mattone posto sotto livello (arretrato di circa una testa) che consente di leggere l'andamento percettivo delle forature nel paramento murario.

Pur non contemplando in questo primo lotto la sistemazione ambientale dell'area adiacente alle mura, rimandata a un secondo lotto di intervento (riguardante sistemazione delle aree a verde e drenaggio delle acque, pavimentazioni esterne, formazione di impianti di illuminazione, di irrigazione e di recinzione perimetrale), il progetto esecutivo ha previsto la creazione di una "zona di rispetto" da realizzare attraverso una fascia perimetrale in ghiaia per evitare il contatto diretto dell'attuale tappeto erboso con la muratura (Figg. 19, 20).

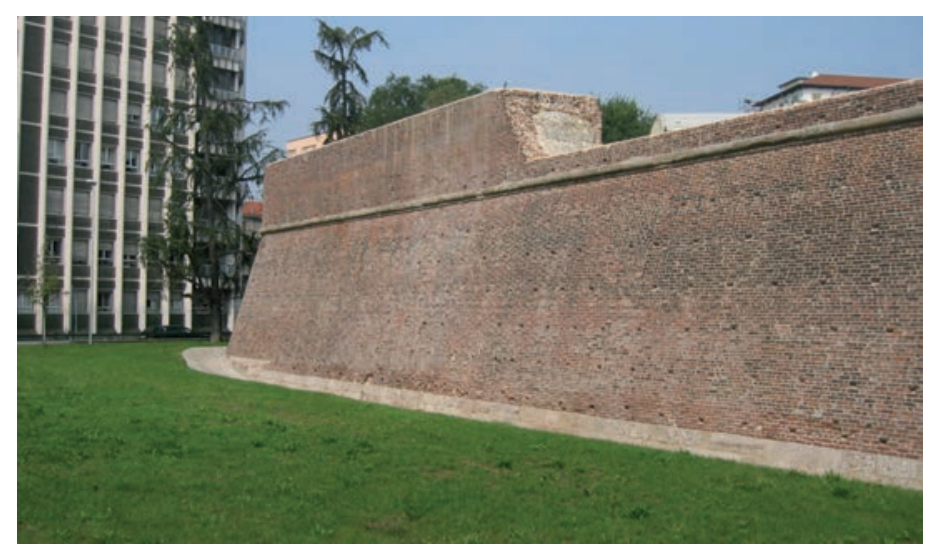

Fig. 19 - Fotografia del bastione al termine dei lavori (2009). 


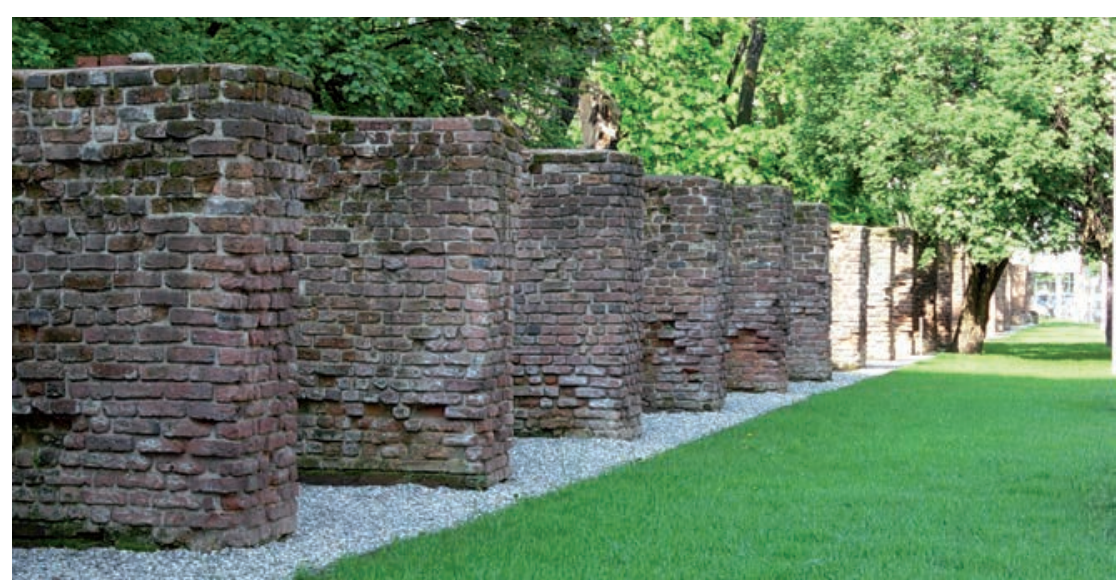

Fig. 20 - Fotografia del tratto T3 di viale Filippetti al termine dei lavori (2009).

\section{CONCLUSiOni}

Le Mura Spagnole sono un complesso monumentale all'aperto e l'ambiente è costituito da uno spazio prevalentemente naturale. Di conseguenza la problematica si identifica con il controllo dello sviluppo dei biodeteriogeni presenti. Infatti sia gli spazi circostanti sia le strutture sono progressivamente interessate dal fenomeno dell'avanzare o del ritorno di tutte le forme di vita a suo tempo presenti; tale processo naturale, si rivela dannoso ai fini della conservazione del patrimonio storico-artistico.

Per la problematica della vegetazione sulle Mura, considerato il legame che si è venuto a creare tra opera di natura e opera dell'uomo, un corretto intervento sulla vegetazione dovrebbe prevedere delle fasi successive: "correzione, conversione e mantenimento" ${ }^{17}$ La prima fase è finalizzata all'eliminazione della vegetazione pericolosa per il manufatto, attraverso l'uso di biocidi, la seconda si propone di agire tempestivamente durante l'eventuale ripresa vegetativa, attraverso ulteriori trattamenti chimici, e l'ultima fase riguarda un programma di manutenzione da condurre attraverso il monitoraggio costante. In tale ottica l'intervento e il

17 BENENTE M., MATTONE M., "Il verde e il rudere: un legame indissolubile”, in Atti del convegno Le Mura di Lucca, Lucca 17-19 maggio 2001, GIUSTI M.A. (a cura di), Alinea, Firenze, 2005, p. 210. 
progetto di manutenzione sono da intendersi come un fatto unitario che si allontana dall'idea dell'intervento di restauro come evento eccezionale, volto a ricondurre l'opera a uno stato "definitivo", e afferma invece l'idea di un processo di cura costante che tende a garantire la più lunga permanenza, regolando le modificazioni che inevitabilmente sono il risultato del divenire. Da una fase di "correzione" prevista con l'intervento di restauro si sarebbe dovuto passare a una fase di "mantenimento". Ad esempio, alla fase di eliminazione della vegetazione considerata pericolosa per il manufatto (trattamento chimico e meccanico della vegetazione superiore), si sarebbe dovuto far seguire un monitoraggio delle specie e una verifica del quadro fessurativo, con eventuale successiva eliminazione degli infestanti nuovamente insediati.

Perché sono state restaurate le Mura spagnole se a otto anni dall'inizio del restauro del primo tratto delle mura e cinque anni dalla conclusione dei lavori ci si trova di fronte alla negazione di una loro cura? Il tempo che è testimone di memorie e monumenti, nel caso delle Mura ha documentato un fare e un abbandono. Lo stato di abbandono in cui versano i tratti di muratura e le frequentazioni di "giardini" e tratti pubblici confermano che non basta ri-pensare la manutenzione in un'ottica di visione processuale della conservazione, ma si deve applicare questa strategia condivisa di sviluppo della conservazione nei termini più ampi possibili, costituita dalle fasi di prevenzione, restauro, manutenzione e gestione del bene, ove l'attività di studio e valorizzazione sono il collante del processo.

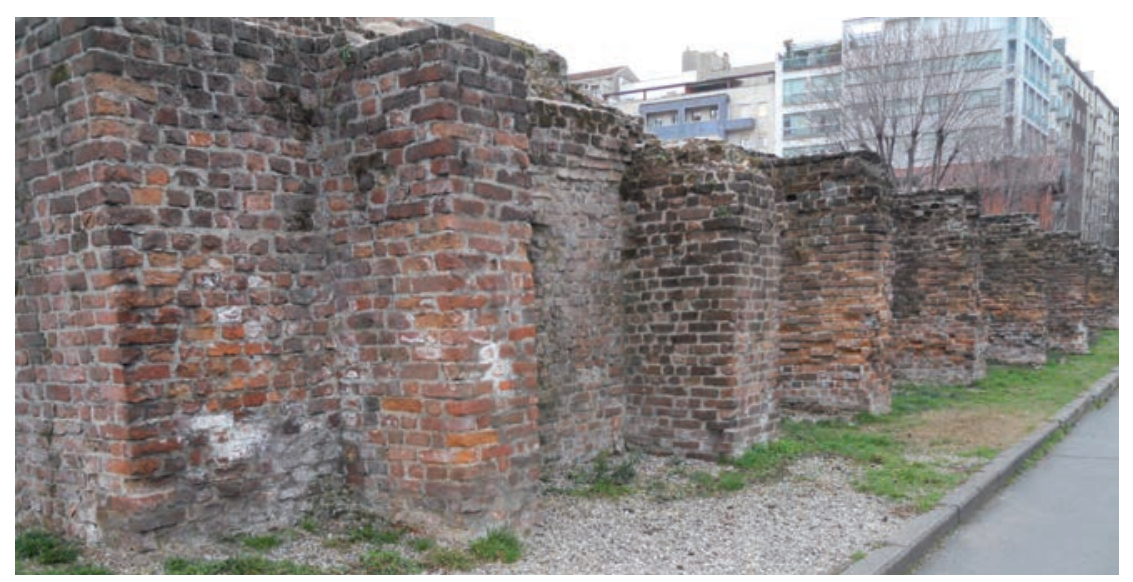

Fig. 21 - Fotografia del tratto T3 di viale Filippetti nel 2012. 
Negli anni del restauro si sono realizzate diverse iniziative culturali da parte dei professionisti coinvolti e della società di sponsorizzazione: convegni e visite guidate dedicate ai cittadini, agli studenti universitari e post laurea, a stranieri, ad associazioni italiane sui castelli, ma nel caso delle Mura non è esistita la volontà di gestione pubblica delle aree, peraltro sancito anche nell'art. 9 della Costituzione italiana (Fig. 21).

\section{BIBLIOGRAFIA}

Di seguito si riporta una breve bibliografia a cui si è fatto riferimento nella Relazione storica del progetto.

G. MAZENTA, Apparato fatto dalla città di Milano per ricevere la serenissima Regina $D$. Margarita d'Austria sposata al potentiss. Re di Spagna D. Filippo III nostro signore, Milano, nella stamperia del q. Pacifico Pontio, 1598 (seconda edizione 1599).

S. LATUADA, Descrizione di Milano, II, Milano 1738.

SCOTTI, Per un profilo dell'architettura milanese (1535-1565), in Omaggio a Tiziano. La cultura artistica milanese nell' età di Carlo V, Catalogo della mostra, Milano 1977.

B.G. VIGOTTI, Impronte romane, medioevali e moderne sull'area dell'Istituto Gaetano Pini, Milano 1981.

PELLEGRINO, Porta Romana, Libreria Milanese, Milano 1985.

S. LEYDI, Le cavalcate dell'ingegnero. L'opera di Gianmaria Olgiati, ingegnere militare di Carlo V, Modena-Ferrara 1989.

A. LANZA, M. SOMARE, Porta Orientale, Romana e Ticinese, Libreria Milanese, Milano 1992.

G. RICCI, "Et parea che Milano diuenuto fosse di tutto il Mondo amplissimo Theatro... ". Festa e teatro a Milano negli ultimi anni del Cinquecento, in La fabbrica, la critica, la storia. Scritti in onore di Carlo Perogalli, a cura di G. COLMUTO ZANELLA, F. CONTI, V. HYBSCH, Milano 1993.

M.T. FIORIO, "Opus turrium et portarum": le sculture di Porta Romana, in Milano e la Lombardia in età comunale. Secoli XI-XIII, Catalogo della mostra, Milano 1993;

S. FROMMEL, Sebastiano Serlio architetto, Milano 1998;

G. GENTILE, D. FEA BIANCOLINI, Chiesa di Santa Maria di Loreto (Santa Marta), in Arona sacra: l'epoca dei Borromeo, catalogo della mostra di Arona, a cura di G. ROMANO, Torino 1977.

Guida d'Italia del Touring Club Italiano, Milano, Milano 1998.

A. SCOTTI, N. SOLDINI, Borromini milanese, in Il giovane Borromini : dagli esordi a San Carlo alle quattro fontane, catalogo della mostra di Lugano, a cura di M. KAHN-ROSSI e M. FRANCIOLLI, Milano 1999. 
V. MILANO, I fratelli Mazenta negli episcopati di Gaspare Visconti e Federico Borromeo, in "Arte lombarda", 131, 2001. F. MINCHIOTTI, Un precedente del pronao di Santa Maria Nuova di Abbiategrasso: Tolomeo Rinaldi e il progetto per l'oratorio di Sant'Agostino a Treviglio, in "Arte lombarda", 131, 2001.

A. MORANDOTTI, Milano profana nell'età dei Borromeo, Milano 2005.

P. VENTRONE, F. MARCHESI, Il passaggio di Margherita d'Austria a Milano tra lutto e festeggiamenti d'occasione, in atti delle giornate di studio Il viaggio attraverso l'Italia di Margherita d'Austria, regina di Spagna, 1598-1599, S. Giuliano Terme (Pisa), 22-23 settembre 2006, (all'epoca in corso di stampa).

\section{Fonti Documentarie}

Archivio Storico del Comune di Milano (ASCM), Località milanesi, 281.

Archivio di Stato di Milano (ASMi), Fondi camerali p.a., 256.

Archivio della Soprintendenza dei Beni Architettonici e del Paesaggio di Milano (AS BB AA PP Mi), cartella E/5/160.

Archivio Borromeo Isola Bella, Chiese nei territori dalla A alla B, Arona.

Biblioteca Ambrosiana di Milano (BAM), S 131 Sup., LVI-LVII e S 132 Sup., CXXIX.”"

Di seguito si riporta una breve bibliografia a cui si è fatto riferimento nella parte del degrado e dell'intervento.

G. CANEVA e G. DE MARCO, Il controllo della vegetazione nelle zone archeologiche e monumentali, in Manutenzione e conservazione del costruito tra tradizione ed innovazione-Bressanone, 1986, pp. 553-569.

AA.VV., Le Pietre nell'Architettura: Struttura e Superfici, Atti del Convegno di Bressanone 25-28 Giugno 1991.

AA.VV., Le Superfici dell'Architettura: il cotto. Caratterizzazioni e trattamenti, Atti del Convegno di Bressanone, 30/6 - 3/7, 1992.

L. MASETTI BITELLI (a cura di), Archeologia, recupero e conservazione, Fiesole, 1993.

G. GOVI, M. SPERANZA, A. CIARALLO, Archeologia e botanica. Ministero per i Beni Culturali e Ambientali, Soprintendenza archeologica di Pompei, 2000.

M.R. DI FABIO a cura di, Le Mura di Ferrara. Storia di un restauro, Minerva Edizioni, Ferrara, 2003.

G.CANEVA, La biologia vegetale per i beni culturali, conoscenza e valorizzazione. Vol II, Nardini Editore, 2001.

M.A. GIUSTI a cura di, Atti del convegno Le Mura di Lucca, Lucca 17-19 maggio 2001, Alinea, Firenze, 2005. 